\title{
Multilevel Noncontiguous Spinal Fractures: Surgical Approach towards Clinical Characteristics
}

\author{
Mehmet Seçer ${ }^{1}$, Fatih Alagöz ${ }^{2}$, Ozhan Uçkun ${ }^{3}$, Oğuz Durmuş Karakoyun ${ }^{2}$, \\ Murat Ömer Ulutaş ${ }^{1}$, Ömer Polat ${ }^{4}$, Ergün Dağlıŏlu ${ }^{2}$, Ali Dalgıç ${ }^{2}$, Deniz Belen ${ }^{2}$ \\ ${ }^{1}$ Neurosurgery Clinic, Sanko University, School of Medicine, Gaziantep, Turkey \\ ${ }^{2}$ Neurosurgery Clinic, Ankara Numune Research and Training Hospital, Ankara, Turkey \\ ${ }^{3}$ Neurosurgery Clinic, Eskisehir Yunus Emre State Hospital, Eskişehir, Turkey \\ ${ }^{4}$ Neurosurgery Clinic, Fatma Hatun Private Hospital, Bolu, Turkey
}

Study Design: The study retrospectively investigated 15 cases with multilevel noncontiguous spinal fractures (MNSF).

Purpose: To clarify the evaluation of true diagnosis and to plane the surgical treatment.

Overview of Literature: MNSF are defined as fractures of the vertebral column at more than one level. High-energy injuries caused MNSF, with an incidence ranging from $1.6 \%$ to $16.7 \%$. MNSF may be misdiagnosed due to lack of detailed neurological and radiological examinations.

Methods: Patients with metabolic, rheumatologic diseases and neoplasms were excluded. Despite the presence of a spinal fracture associated clearly with the clinical picture, all patients were scanned within spinal column by direct X-rays, computed tomography and magnetic resonance imaging. When there were $\geq 5$ intact vertebrae between two fractured vertebral segments, each fracture region was managed with a separated stabilization. In cases with $\leq 4$ intact segments between two fractured levels, both fractures were fixed with the same rod and screw system.

Results: There were 32 vertebra fractures in 15 patients. Eleven (73.3\%) patients were male and age ranged from 20 to 64 years

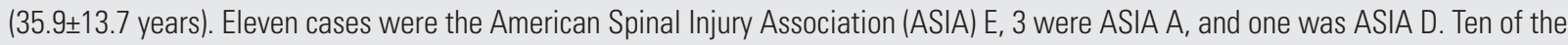
$15(66.7 \%)$ patients returned to previous social status without additional deficit or morbidity. The remaining $5(33.3 \%)$ patients had mild or moderate improvement after surgery.

Conclusions: The spinal column should always be scanned to rule out a secondary or tertiary vertebra fracture in vertebral fractures associated with high-energy trauma. In MNSF, each fracture should be separately evaluated for decision of surgery and planned approach needs particular care. In MNSF with $\leq 4$ intact vertebra in between, stabilization of one segment should prompt the involvement of the secondary fracture into the system.

Keywords: Spinal column; Spinal fracture; Multilevel; Surgery

\section{Introduction}

Multilevel noncontiguous spinal fractures (MNSF) are rare and have unique characteristics. MNSF are defined as injuries to the vertebral column at more than one site and separated by several normal vertebrae [1-7]. There must be at least 3 normal vertebrae between the fractured levels by definition in the literature though a single normal ver-

Received Apr 07, 2015; Accepted Apr 23, 2015

Corresponding author: Ali Dalgiç

Neurosurgery Clinic, Ankara Numune Research and Training Hospital,

B-Blok, Kat: 3 06100, Altindag, Ankara, Turkey

Tel: +90-505-234-2662, Fax: +90-312-311-4340, E-mail: alidalgic@yahoo.com 
tebra is also acceptable by several authors [3-5]. The reported incidence of noncontiguous spinal fractures varies from $1.6 \%$ to $16.7 \%$, with a usual range from $3 \%$ to $8 \%$ [17]. MNSF may present with local pain, paraplegia, quadriplegia, or even death $[1,2,4]$. Except for the fractures due to osteoporosis, they usually occur as a consequence of high-energy trauma $[1,2,8]$.

Rapid diagnosis of MNSF is essential since a misdiagnosis or delayed diagnosis may complicate the clinical picture. A fracture at a secondary or tertiary region, as a part of MNSF, might cause and/or aggravate neurological deficit, spinal instability, deformity and need for additional surgical intervention $[3,6,9,10]$. In this study, patients with at least 1 intact vertebra between the fractured vertebrae were investigated with respect to demographic properties, clinical and radiological findings, trauma mechanism, treatment approach, and clinical outcomes.

\section{Materials and Methods}

This study retrospectively examined 15 patients who presented to neurosurgery clinics of two different centers and underwent surgical treatment for MNSF between 2012 and 2014. Patients having metabolic, rheumatologic diseases, and neoplasms such as osteoporosis, ankylosing spondylitis, and multiple myeloma were excluded. Patients treated with conservative measures for a single fracture level or levels were also excluded. Fractures involving the occipitocervical junction and sacrum were also excluded since they have a have a unique anatomy, biomechanics, and classification.

All cases were considered as systemic multi-trauma and further investigations were done for associated cranial, thoracic, abdominal, and extremity lesions. Although a fracture was found to be clearly associated with the neurological signs of all patients, direct X-ray examinations involving cervical, thoracic, lumbar regions as well as detailed extremity studies were obtained in all patients. Thorax and abdominal computed tomography (CT) imaging was performed as a routine procedure in high energy multitrauma patients. Additional fractures might be visualized during these investigations if bone scans were thoroughly evaluated Apart from these investigations at presentation, CT and magnetic resonance imaging (MRI) studies were also performed for confirmatory diagnosis, treatment indication or surgical planning.

Cases with unstable vital signs including blood pressure, blood oxygen level, and serum hemoglobin level were precisely investigated for an associated systemic problem nevertheless primary measures were immediately performed to correct vital signs. Cases with stable vital signs were operated on an elective emergency basis. Fractures of all patients were stabilized at a single stage. Symptomatic vertebral fractures with overt neurological

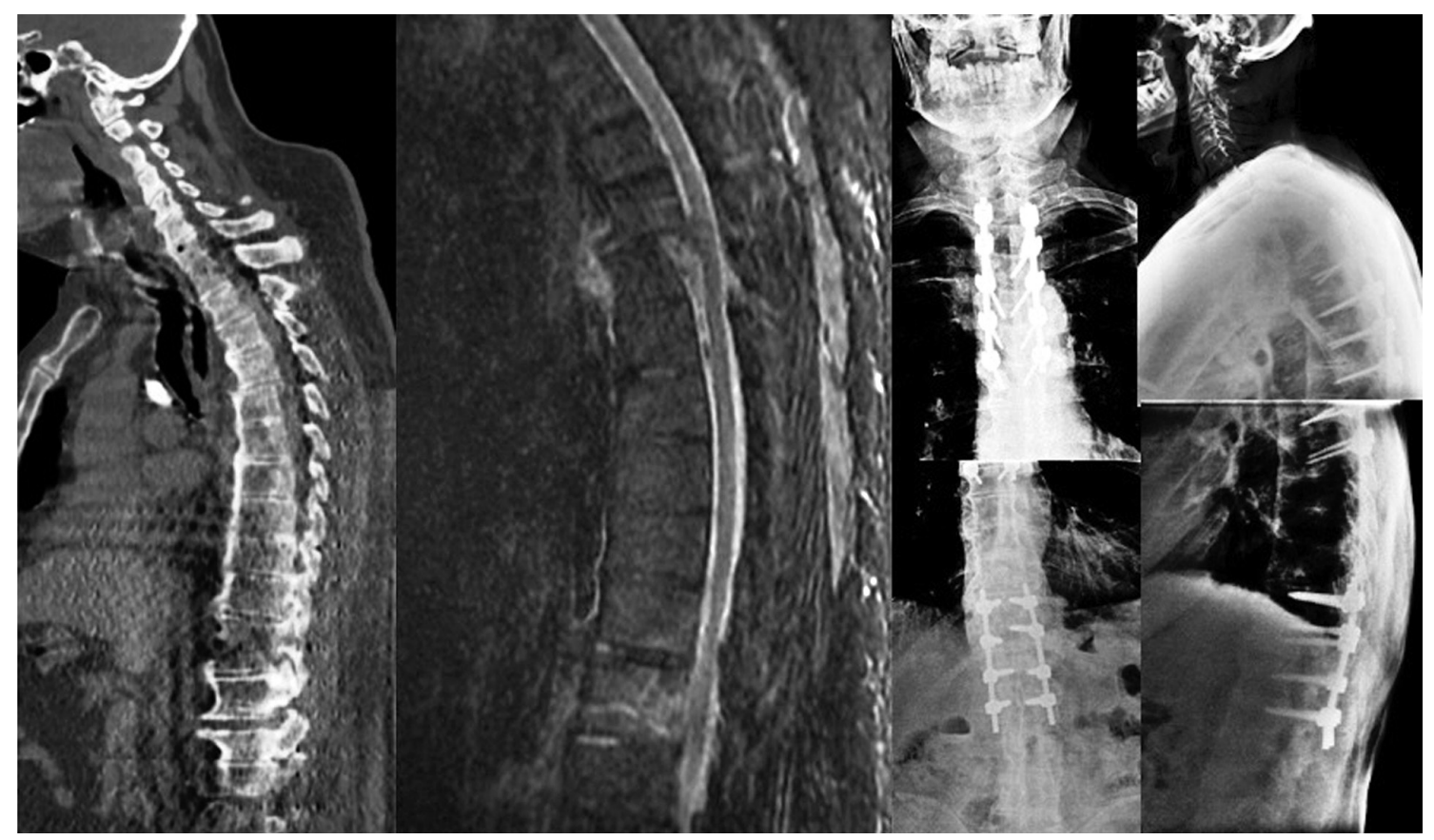

Fig. 1. Separate stabilization systems applied for ankylosing spondylitis with T3 and T12 fractures. 
signs were primarily operated on, whether the localization of fracture was proximal or distal. In the remaining cases without an associated neurological deficit, proximal segment was stabilized first. When there were $\geq 5$ intact vertebrae between two fractured vertebral segments, each fracture region was managed with a separate operative incision and approach for stabilization (Fig. 1). In cases with $\leq 4$ intact segments between two fractured levels, both fractures were fixed with the same rod and screw system (Fig. 2).

\section{Results}

Eleven $(73.3 \%)$ patients were male and 4 (26.7\%) were female. Age ranged between 20 and 64 years $(35.9 \pm 13.7$ years). The most common mechanism of accident resulting in spinal fractures was falling down from height in 7 (46.6\%) patients, followed by traffic accidents in $6(40 \%)$ and motorcycle accidents in 2 (13.3\%). Associated injuries were diagnosed in 6 patients and documented as 1 bilateral hemothorax, 1 left-sided pneumothorax, 2 right-sided pneumothorax, 1 calcaneus fracture and 1 tibia fracture. Vertebral fractures involving three segments were noted in 2 cases whereas two segments were involved in 13 cases with a minimum one or more single intact vertebral

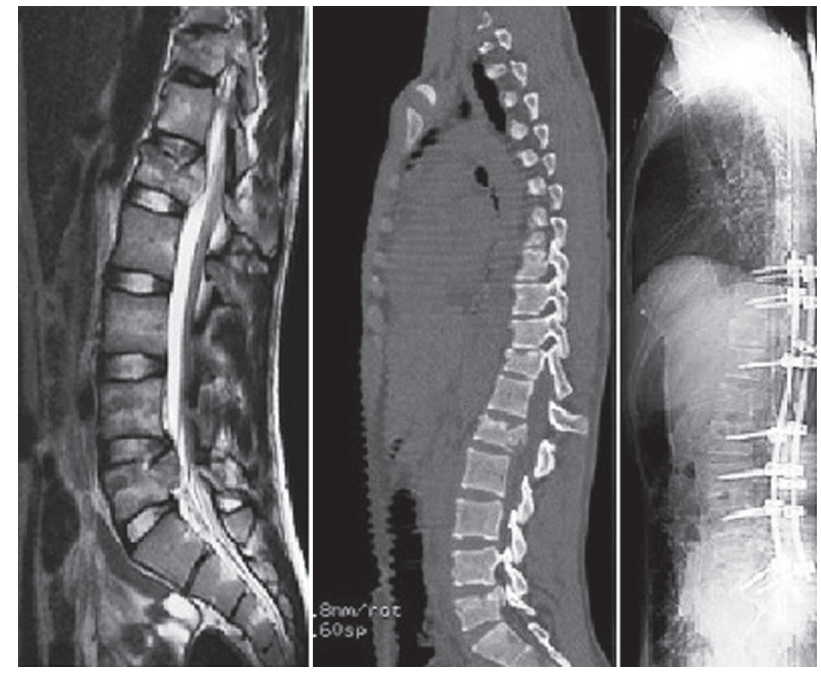

Fig. 2. Magnetic resonance imaging revealing expansion of fractures into the spinal canal at the levels of Th11-L1-L5 vertebrae that were stabilized by a single fixation system.

segment in between. There were 32 vertebral fractures. The most common segment among all patients suffering MNSF was thoracic+thoracic region (40\%), followed by thoracic+lumbar region (26.7\%), lumbar+lumbar region (20\%), and cervical+lumbar region (13.3\%). According to the American Spinal Injury Association (ASIA) classification, 11 cases had ASIA E, 3 had ASIA A, and 1 had ASIA

Table 1. Demographic conditions and distribution of the fractures according to AO classification

\begin{tabular}{|c|c|c|c|c|c|c|c|}
\hline $\begin{array}{l}\text { Case } \\
\text { no. }\end{array}$ & $\begin{array}{l}\text { Age } \\
(y r)\end{array}$ & Sex & Injury & ASIA & $\begin{array}{l}\text { Level-A0 } \\
\text { classification }\end{array}$ & $\begin{array}{l}\text { Stabilized } \\
\text { segments }\end{array}$ & Additional injury \\
\hline 1 & 24 & Female & Traffic accident & $E$ & Th10 $\rightarrow$ A1, Th12 $\rightarrow A 2, L 1 \rightarrow A 3$ & Th8-L2 & - \\
\hline 2 & 50 & Male & Traffic accident & $\mathrm{E}$ & Th6 $\rightarrow$ C1, Th9 $\rightarrow$ C2 & Th3-11 & Calcaneus fracture \\
\hline 3 & 47 & Female & Falling & E & Th12 $\rightarrow$ C2, L3 $\rightarrow$ A3 & Th10-L5 & - \\
\hline 4 & 42 & Male & Falling & $\mathrm{E}$ & $\mathrm{L} 3 \rightarrow \mathrm{B} 2, \mathrm{~L} 5 \rightarrow \mathrm{B} 2$ & L2-S1 & Tibia fracture \\
\hline 5 & 24 & Male & Falling & E & $L 3 \rightarrow B 1, L 5 \rightarrow B 3$ & L2-S1 & - \\
\hline 6 & 24 & Male & Traffic accident & $\mathrm{E}$ & Th1 $\rightarrow$ A1, L1 $\rightarrow B 3$, L5 $\rightarrow B 2$ & Th10-S1 & Bilateral hemothorax \\
\hline 7 & 30 & Female & Falling & E & $\mathrm{L} 1 \rightarrow \mathrm{A} 3, \mathrm{~L} 5 \rightarrow \mathrm{A} 3$ & T11-S1 & Right pneumothorax \\
\hline 8 & 35 & Male & Traffic accident & A & Th5 $\rightarrow$ C1, Th8 $\rightarrow$ C2 & Th2-Th12 & - \\
\hline 9 & 20 & Male & Falling & $\mathrm{E}$ & Th6 $\rightarrow$ B2, Th8 $\rightarrow$ B3 & Th4-Th10 & - \\
\hline 10 & 64 & Male & Traffic accident & $E$ & Th4 $\rightarrow A 2$, Th10 $\rightarrow A 2$ & Th2-T12 & - \\
\hline 11 & 35 & Male & Falling & D & Th11 $\rightarrow$ C2, L1 $\rightarrow$ C3 & Th9-L3 & Bilateral pneumothorax \\
\hline 12 & 21 & Male & Motorcycle accident & A & $\mathrm{C} 7 \rightarrow \mathrm{C} 1, \mathrm{~L} 1 \rightarrow \mathrm{A} 3$ & C6-Th1, Th12-L2 & Right pneumothorax \\
\hline 13 & 48 & Female & Traffic accident & $E$ & Th5 $\rightarrow$ B2, Th8 $\rightarrow$ B3 & Th3-Th10 & - \\
\hline 14 & 52 & Male & Falling & E & Th4 $\rightarrow \mathrm{C} 2$, Th7 $\rightarrow \mathrm{C} 2$ & Th2-Th9 & - \\
\hline 15 & 23 & Male & Motorcycle accident & A & $\mathrm{C} 6 \rightarrow \mathrm{C} 3, \mathrm{~L} 1 \rightarrow \mathrm{A} 3$ & C4-C7, Th12-L2 & - \\
\hline
\end{tabular}

ASIA, American Spinal Injury Association. 
$\mathrm{D}$ at primary neurological evaluation. The exact levels of fractures and their distribution according to the $\mathrm{AO}$ spinal fracture classification and neurological status are summarized in Table 1.

Posterior stabilization was performed in all cases and an additional decompressive measure was done when necessary. None of the patients developed an additional postoperative neurological deficit. The level of the stabilization was determined by using the AO spinal fracture classification (McCormack et al. [11]). The main aim of using two systems in patients with $\geq 5$ intact segments between fractured levels was to evenly distribute the available load and thereby prevent overloading of the system for kyphotic deformity.

ASIA class did not improve in any patient during the early postoperative period. Ten of 15 (66.7\%) patients returned to previous social status without additional deficit or morbidity however 5 (33.3\%) patients had mild or moderate improvement after surgery.

\section{Discussion}

MNSF is defined as multilevel fractures involving nonneighboring vertebrae. In the present study, we reviewed the clinical and radiological features of MNSF and emphasized that a secondary fracture might be overlooked when there is a symptomatic primary fracture explaining the present clinical findings. Furthermore a surgical approach towards these fractures should be combined when there are $\leq 4$ intact vertebral segments in between.

The primary lesion is the major lesion that is responsible from clinical signs and symptoms. Major vertebral fracture is easily recognized clinically or radiologically, but an associated secondary or tertiary fracture might pose a diagnostic challenge in some situations. This diagnostic dilemma is more pronounced when the symptomatic lesion is proximal to the secondary and tertiary fracture. In particular, when a patient presents with symptoms of paraplegia and an associated cervical fracture, a secondary thoracic or lumbar fracture might be easily be overlooked. Diagnosis of a secondary fracture may be delayed as long as 2.8 to 52.6 days in the literature $[1,7]$. Lian et al. [2] reported a delay of 5.1 days for diagnosis of a secondary fracture in 8 of 30 patients. Delays in diagnosis of such lesions have been explained by focusing on a particular lesion indicated by neurological signs $[1,7]$ and the inability to evaluate advanced radiological imaging tests during management of additional traumatic pathologies including hemothorax and cerebral contusion [6].

Neurological examination is the key guide for lesion localization in traumatic lesions of the spinal vertebrae. A great majority of the cases in our series had complete neurological injury or normal findings on examination. Thus, general clinical picture might not be so helpful for detection of a secondary fracture in the present series. In this study, 15 patients were evaluated for a secondary fracture and majority of patients did not have any neurological deficit. Clinical picture did not help in the diagnosis of a secondary lesion. On the other hand two cases in the present study were reported to have a fixed neurological deficit (ASIA A) and an associated fracture distal to the primary lesion was put into shadow. Hence, radiological examinations have a more important role in diagnosis of a secondary or tertiary fracture. MNSF incidence is as high as $20 \%$ and Calenoff et al. [7] reported that a secondary lesion occurs above the primary lesion in $40 \%$ of cases and below the primary lesion in $60 \%$ of cases. For this reason, all vertebrae should be carefully examined as routine when there are signs of a single segment vertebra fracture in high-energy traumas.

We suggest that not only the neighboring vertebrae should be examined, but also that the whole vertebral column should be evaluated for presence of an associated fracture. All patients in our series were diagnosed as MNSF with the aid of a radiological investigation even when there were no neurological signs. Similarly, both proximal and distal segments of the lesion should be examined in cases presenting with complete spinal injuries. A delay in the diagnosis of a secondary or even a tertiary lesion is important due to a risk of an additional neurological deficit, spinal instability, deformity, and planning of surgical intervention for primary fracture level $[5,7]$. Thus, precise evaluation of whole vertebral column with CT or MRI is essential to avoid an incomplete diagnosis in patients with a spinal vertebra fracture.

Key procedures in the surgical management of vertebra fractures include decompression of neural elements and restoration of vertebral alignment. Early surgical stabilization shortens the hospital stay, early rehabilitation, and reduces rates of complications due to prolonged bed-rest, such as pneumonia, decubitus ulcers, and muscle atrophy [8-10]. Treatment strategy for MNSF and related surgical complications are not different than single level fractures. Lian et al. [2] compared three treatment modalities of 
conservative treatment, surgical therapy for a single lesion and surgical therapy for both lesions in a vertebral MNSF series of 30 patients and found the best clinical and radiological outcome in the surgically managed group [3]. Jorgensen and Joseph [8] reported that excessive kyphosis and resultant chronic pain developed in a case with Th11 and L2 compression fractures, since they were not able to manage surgically due to severe infectious findings. In the present series early surgical approach provided early mobilization at every patient, which prevented prospective systemic complications due to immobility.

Posterior stabilization systems become more popular in the last decade as a gold standard technique. Anterior decompression and stabilization is usually inadequate to provide a biomechanically strong system and surgical approach is associated with more complications than the posterior stabilization procedures [12]. Posterior stabilization systems can restore vertebral body height by distraction forces. Furthermore, anterior and middle columns maintained their normal length during correction of kyphosis. Distractive forces provided by the posterior stabilization system developed a tensile strength in the posterior longitudinal ligament which pushes back the retropulsed bone fragments forward. This process has been termed ligamentotaxis and it is beneficial particularly if performed at the early period [13].

Spinal fractures associated with MNSF should be evaluated as separate in terms of treatment approach. Posterior approaches for stabilization are generally preferred for MNSF. It is a conventional method for the management of vertebral fractures with low complication rates. McCormack et al. [11] suggested a load sharing system for vertebral fractures taking the level of injury at horizontalvertical planes and angle of kyphosis at the level of fracture to consideration. The stabilization system failed and the screws were reported to be broken in burst fractures subjected to short segment fixation (one level above and below the fracture segment). Despite the presence of an increased intention towards short segment stabilization in recent years, the complications of anterior approach should not be overlooked. A meta-analysis demonstrated the association of the anterior approach with longer operation time and greater blood loss. On the other hand, anterior support can also be provided by placing cages and/or grafts via extended posterolateral approaches [14]. In the present study, the anterior approach was not performed for vertebral fractures and posterior decom- pression with long segment stabilization was preferred to obtain an adequate alignment.

The evaluation of the number of intact vertebrae between two fractured segments is also critical in deciding the treatment approach for MNSF in addition to neurological deficit, spinal deformity, and instability. The length of the system used for the stabilization of the primary fracture causing neurological picture should not jeopardize the secondary and/or tertiary fractures. The expected junctional kyphosis, especially at the proximal part of the stabilized segment, is a well-known complication in vertebral fractures. Proximal junctional kyphosis has been reported at a rate as high as $26 \%$ and is more common in the thoracic region after corrective surgery for long segment vertebral deformities [15]. A caudal junctional kyphosis might also rarely develop distal to the stabilization system [16]. A biomechanical overload on the secondary fracture should not be overlooked since a stabilization system involving the primary fracture might jeopardize the secondary fracture when there are fewer than 2 intact vertebrae in between. To our knowledge, the length of the stabilization system needs to be precisely planned to distribute biomechanical loading and to preserve the present mechanics of the already fractured secondary lesion.

\section{Conclusions}

The spinal column should be scanned to rule out a secondary or tertiary vertebra fracture in vertebral fractures due to high-energy trauma and severe injury. In MNSF, each fracture should be separately evaluated for decision of surgery and planned approach needs particular care. In MNSF with $\leq 4$ intact vertebrae in between, stabilization of one segment should prompt the involvement of the secondary fracture into the system.

\section{Conflict of Interest}

No potential conflict of interest relevant to this article was reported.

\section{References}

1. Korres DS, Boscainos PJ, Papagelopoulos PJ, Psycharis I, Goudelis G, Nikolopoulos K. Multiple level noncontiguous fractures of the spine. Clin Orthop Relat Res 2003;(411):95-102. 
2. Lian XF, Zhao J, Hou TS, Yuan JD, Jin GY, Li ZH. The treatment for multilevel noncontiguous spinal fractures. Int Orthop 2007;31:647-52.

3. Korres DS, Katsaros A, Pantazopoulos T, Hartofilakidis-Garofalidis G. Double or multiple level fractures of the spine. Injury 1981;13:147-52.

4. Iencean SM. Double noncontiguous cervical spinal injuries. Acta Neurochir (Wien) 2002;144:695-701.

5. Powell JN, Waddell JP, Tucker WS, Transfeldt EE. Multiple-level noncontiguous spinal fractures. J Trauma 1989;29:1146-50.

6. Wittenberg RH, Hargus S, Steffen R, Muhr G, Botel U. Noncontiguous unstable spine fractures. Spine (Phila Pa 1976) 2002;27:254-7.

7. Calenoff L, Chessare JW, Rogers LF, Toerge J, Rosen JS. Multiple level spinal injuries: importance of early recognition. AJR Am J Roentgenol 1978;130:665-9.

8. Jorgensen DR, Joseph J Jr. Multiple noncontiguous spine fractures at four levels in a neurologically intact patient. J Trauma 1996;41:750-3.

9. Acaroglu ER, Alanay A. Four-level noncontiguous fracture of the vertebral column: a case report. J Orthop Trauma 2001;15:294-9.

10. Guo HG, Ma XL, Li FT, Feng SQ. Five-level noncontiguous spinal injuries of cervical region: report of a case and literature review. Chin Med J (Engl) 2012; 125:2777-80.

11. McCormack T, Karaikovic E, Gaines RW. The load sharing classification of spine fractures. Spine (Phila Pa 1976) 1994;19:1741-4.

12. Lin B, Chen ZW, Guo ZM, Liu H, Yi ZK. Anterior approach versus posterior approach with subtotal corpectomy, decompression, and reconstruction of spine in the treatment of thoracolumbar burst fractures: a prospective randomized controlled study. J Spinal Disord Tech 2011 Jun 1 [Epub]. http://dx.doi. org/10.1097/BSD.0b013e3182204c53.

13. Aebi M, Etter C, Kehl T, Thalgott J. Stabilization of the lower thoracic and lumbar spine with the internal spinal skeletal fixation system. Indications, techniques, and first results of treatment. Spine (Phila $\mathrm{Pa}$ 1976) 1987;12:544-51.

14. Dalgic A, Uckun O, Acar HI, et al. Single-stage posterolateral Corpectomy and circumferential stabilization without laminectomy in the upper thoracic spine: cadaveric study and report of three cases. Turk Neurosurg 2010;20:231-40.

15. Glattes RC, Bridwell KH, Lenke LG, Kim YJ, Rinella A, Edwards C, 2nd. Proximal junctional kyphosis in adult spinal deformity following long instrumented posterior spinal fusion: incidence, outcomes, and risk factor analysis. Spine (Phila Pa 1976) 2005;30:1643-9.

16. Kwon BK, Elgafy H, Keynan O, et al. Progressive junctional kyphosis at the caudal end of lumbar instrumented fusion: etiology, predictors, and treatment. Spine (Phila Pa 1976) 2006;31:1943-51. 